\title{
Can Diffusion Tensor Imaging Noninvasively Detect IDH1 Gene Mutations in Astrogliomas? A Retrospective Study of 112 Cases
}

\author{
W.L. Tan, W.Y. Huang, B. Yin, J. Xiong, J.S. Wu, and D.Y. Geng
}

\begin{abstract}
BACKGROUND AND PURPOSE: IDH1 mutational status probably plays an important role in the predictive response for patients with astroglioma. This study explores whether DTI metrics are able to noninvasively detect IDH1 status in astrogliomas.

MATERIALS AND METHODS: The DTI data of 112 patients with pathologically proven astroglioma (including 25, 12 , and 10 cases with IDH1 mutation and 11, 11, and 43 cases without mutation in grades II, III, and IV, respectively) were retrospectively reviewed. The maximal fractional anisotropy, minimal ADC, ratio of maximal fractional anisotropy, and ratio of minimal ADC in the tumor body were measured. In the same World Health Organization grading, the imaging parameters of patients with and without IDHI RI32H mutation were compared by means of optimal metrics for detecting mutations. Receiver operating characteristic curve analysis was performed.

RESULTS: The maximal fractional anisotropy and ratio of maximal fractional anisotropy values had statistical significance between patients with IDH7 R132H mutation and those without mutation in astrogliomas of grades II and III. The areas under the curve for maximal fractional anisotropy and ratio of maximal fractional anisotropy were both 0.92 in grade II and 0.80 and 0.82 in grade III. The minimal ADC value and ratio of minimal ADC value also demonstrated statistical significance between patients with mutation and those without mutation in all astroglioma grades. The areas under the curve for minimal ADC were 0.94 (II), 0.76 (III), and 0.66 (IV), and the areas under the curve for ratio of minimal ADC were 0.93 (II), 0.83 (III), and 0.70 (IV).
\end{abstract}

CONCLUSIONS: Fractional anisotropy and ADC from DTI can noninvasively detect IDH1 R132H mutation in astrogliomas.

ABBREVIATIONS: $I D H]=$ isocitrate dehydrogenase $1 ; \mathrm{FA}=$ fractional anisotropy; $r \mathrm{rmFA}=$ ratio of maximal fractional anisotropy; $r \mathrm{mADC}=$ ratio of minimal $\mathrm{ADC}$; $A \cup C=$ area under the curve; $\mathrm{WHO}=$ World Health Organization

M utations in isocitrate dehydrogenase 1 (IDH1) exist in a large percentage of gliomas. ${ }^{1,2}$ The value of detecting IDH1 mutational status has been reported in diagnostic and prognostic studies, and it might also be useful as a predictive indicator. IDH1 mutations are not found in nonneoplastic conditions thus far. ${ }^{3-5}$ Mutations in the IDH1 gene were associated with improved outcomes in patients with anaplastic astrocytomas and glioblastomas. ${ }^{2}$ Furthermore, Juratli et $\mathrm{al}^{6}$ suggested that early radiation therapy appeared to be beneficial only in patients with low-grade astrocytoma with $I D H$ mutations. SongTao et $\mathrm{al}^{7}$ reported that

Received August 1, 2013; accepted after revision October 1 .

From the Departments of Radiology (W.L.T., W.Y.H., B.Y., D.Y.G.), Neuropathology (J.S.X.), and Neurosurgery (J.W.), Huashan Hospital, Fudan University, Shanghai, Peoples Republic of China.

This work was supported by the National Natural Science Foundation of China (81071132) and by the Natural Science Foundation of Shanghai (10411952100).

Please address correspondence to Daoying Geng, MD, No. 12, Middle Rd Wu Lu Mu Qi, Shanghai, PR China; e-mail: gengdy@163.com

- Indicates open access to non-subscribers at www.ajnr.org

http://dx.doi.org/10.3174/ajnr.A3803
IDH mutations were correlated with a higher rate of objective response to temozolomide.

Thus far, the reference standard for testing the status of IDHI is gene sequencing. Immunohistochemical analysis by use of the mouse monoclonal IDH1 R132H antibody is commonly applied in clinical examinations with high sensitivity. ${ }^{8}$ However, both sequencing and immunohistochemical examinations require tumoral tissues, and neither method can offer preoperative detection of IDH1 gene status. Some patients will not or cannot undergo operations (such as patients with brain stem gliomas or with poor physical condition). Therefore, we need a noninvasive method to detect IDH1 mutational status before surgery or biopsy. Metellus et $\mathrm{al}^{9}$ reported morphologic changes in IDH1 mutant gliomas but lack of a quantitative indicator. Glioma-associated IDH1 mutations result in overproduction of the oncometabolite $\mathrm{R}(-)$-2-hydroxyglutarate. ${ }^{10,11}$ Although the concentration of 2-hydroxyglutarate in serum cannot be used to judge the status of IDH1 mutations, these mutations are associated with the accumulation of $\mathrm{R}(-)$-2-hydroxyglutarate within the tumor. ${ }^{12,13}$ Pope et $\mathrm{al}^{14}$ suggested that MR spectroscopy could detect 2-hy- 
droxyglutarate in patients with $I D H 1$-mutant glioma and provide a noninvasive measurement of 2-hydroxyglutarate in gliomas. However, the measurement of 2-hydroxyglutarate through MR spectroscopy is performed by special software and is inconvenient for clinical application.

DTI has been used to detect the genetic characteristics of gliomas. ${ }^{15}$ In the current study, we explore whether the metrics determined from DTI can help to detect IDH1 gene status of astrogliomas noninvasively.

\section{MATERIALS AND METHODS Clinical Data and Groupings}

A total of 112 patients with pathologically proven astroglioma and preoperatively performed DTI were retrospectively reviewed from January 2011 to June 2012 in our hospital. Clinical data, including age and sex, were collected. According to the 2007 World Health Organization (WHO) classification of tumors of the central nervous system, ${ }^{16}$ there were 36 cases of grade II astrocytoma, 23 cases of grade III anaplastic astrocytoma, 46 cases of grade IV primary glioblastoma, and 7 cases of grade IV secondary glioblastoma.

IDH1 R132H immunohistochemical results were also reviewed. IDH1 $\mathrm{R} 132 \mathrm{H}$ protein expression was determined in paraffin-embedded tumor specimens after surgery by use of anti-mIDH1R132H antibody (internal clone H09; Dianova, Hamburg, Germany). The expression of IDH1 R132H was determined by use of the methods of Takano et al. ${ }^{17}$ Cases with $\geq 10 \%$ positive cells were rated positive, and cases with $<10 \%$ positive cells were rated negative. There were 25 (69.44\%), 12 (52.17\%), and $10(18.87 \%)$ cases showing positive results in grades II, III, and IV, respectively. Six of 10 cases of grade IV glioblastoma with positive results were primary glioblastomas.

All of the patients were divided into 6 groups according to immunochemical results and WHO grades as follows: 1) group 1: grade II with IDH1 R132H mutation; 2) group 2: grade II without IDH1 R132H mutation; 3) group 3: grade III with IDH1 R132H mutation; 4) group 4: grade III without IDH1 R132H mutation; 5) group 5: grade IV with IDH1 R132H mutation; and 6) group 6: grade IV without IDH1 R132H mutation.

This study was approved by the local research ethics committee. Patient informed consent was waived for this retrospective study.

\section{MR Technique}

MR scanning was performed with the use of a 3T scanner (MAGNETOM Verio; Siemens, Erlangen, Germany), with an 8-channel head coil. All of the patients underwent preoperative DTI. Parameters were as follows: $\mathrm{TR}=7600 \mathrm{~ms}$; $\mathrm{TE}=91 \mathrm{~ms}$; $\mathrm{FOV}=23.0 \mathrm{~cm} \times 23.0 \mathrm{~cm}$; acquisition matrix of $128 \times 128$; section thickness $=3 \mathrm{~mm}$; no intersection separation; $b=0 / 1000$ seconds $/ \mathrm{mm}^{2}$; number of excitations $=2$; bandwidth $=1502$ $\mathrm{Hz} / \mathrm{Px}$. Twenty directions were acquired. A single-shot echo-planar imaging diffusion tensor sequence was used. Other sequences including T2WI $(\mathrm{TR}=4000 \mathrm{~ms}, \mathrm{TE}=96 \mathrm{~ms}$, number of excitations $=1$, echo trains per section $=8$, bandwidth $=221 \mathrm{~Hz} / \mathrm{Px})$, and T1WI and postcontrast T1WI $(\mathrm{TR}=2000 \mathrm{~ms}, \mathrm{TE}=17 \mathrm{~ms}$, number of excitations $=1$, echo trains per section $=17$, band- width $=260 \mathrm{~Hz} / \mathrm{Px}$ ). Parallel imaging was used in all the sequences mentioned above with acceleration factor $=2$.

\section{Image Analysis}

According to the DTI data, the workstation automatically generated the fractional anisotropy (FA) and ADC mapping. All of the FA and ADC maps were retrospectively interpreted by 2 experienced neuroradiologists (W. Tan and W. Huang, with 10 and 6 years of brain MR imaging experience, respectively) who did not know the pathologic results. The maximal FA was measured by manually placing ROIs in the most solid and highest signal-intensity part of the tumoral regions on the FA mapping by visual inspection. The size of every ROI was between 15-20 pixels. The mean value of small ROIs drawn to encompass the voxels with maximal FA values was recorded. Each neuroradiologist drew 3 ROIs to obtain the maximal FA, according to the gray-scale map. The maximal FA value among these values was chosen as the result. An average of the results of 2 neuroradiologists was used as the patient's maximal FA. If the value for one reader varied by $>20 \%$ from the second reader, ${ }^{18}$ a third reader, a neuroradiologist with 22 years of experience (D. Geng), would measure the maximal FA value. The average of Dr Geng's result and the result closer to her result from the other 2 neuroradiologists was used as the patient's maximal FA, and $22(19.6 \%)$ patients required the third reader method. Cystic and hemorrhage areas were excluded according to T2WI and enhanced T1WI, when the ROI was drawn. The minimal ADC was obtained by the same method, and $17(15.2 \%)$ patients required the third reader method.

The FA and ADC values were also measured in the peritumoral regions. The peritumoral area is defined as a region containing nonenhanced and higher signal intensity outside the tumoral solid area on T2WI and ADC images. If there was not different signal noticed from T2WI and ADC mapping, especially in grade II tumors, the ROI was manually drawn in the peritumoral area adjoining to the tumoral area $(<1 \mathrm{~cm})$. Forty-five $(40.18 \%)$ and $36(32.14 \%)$ patients required the third reader method in the measurement of peritumoral FA and ADC, respectively. Because of regional variation of the FA and ADC absolute values, ${ }^{19}$ the contralateral normal posterior limb of the internal capsule was measured as the control to normalize FA and ADC values. The ratio of maximal FA (rmFA), ratio of peritumoral FA or ratio of minimal ADC (rmADC), and ratio of peritumoral $\mathrm{ADC}$ were equal to the maximal FA, peritumoral FA or minimal $\mathrm{ADC}$, and peritumoral ADC divided by the contralateral FA or contralateral ADC.

\section{Statistics}

All of the statistical analyses were performed with the use of the Statistical Package for the Social Sciences software (version 13.0; IBM, Armonk, New York). The Kolmogorov-Smirnov test was performed on the age and imaging data to analyze whether they were normally distributed. Statistical significance was set at the $P<.05$ level. The interobserver intraclass correlations were calculated for the maximal FA, minimal ADC, peritumoral FA, peritumoral ADC, contralateral FA, and contralateral ADC, respectively. Receiver operating characteristic curves of the parameters with statistical significance were obtained to seek the cutoff value 
Table 1: Results of clinical and imaging data

\begin{tabular}{lcccccc}
\hline & Group 1 & Group 2 & Group 3 & Group 4 & Group 5 & Group 6 \\
\hline No. of patient cases & 25 & 11 & 12 & 11 & 10 & 43 \\
Sex, M/F & $15 / 10$ & $5 / 6$ & $9 / 3$ & $7 / 4$ & $8 / 2$ & $27 / 16$ \\
Age, y & $39.68 \pm 7.22$ & $42.45 \pm 18.38$ & $41.92 \pm 8.71$ & $42.64 \pm 17.90$ & $41.70 \pm 11.39$ & $54.42 \pm 12.63$ \\
Edema FA & $0.26 \pm 0.12$ & $0.28 \pm 0.14$ & $0.18 \pm 0.09$ & $0.24 \pm 0.11$ & $0.18 \pm 0.06$ & $0.20 \pm 0.09$ \\
Ratio of edema FA & $0.37 \pm 0.17$ & $0.40 \pm 0.20$ & $0.24 \pm 0.12$ & $0.34 \pm 0.16$ & $0.25 \pm 0.07$ & $0.27 \pm 0.13$ \\
Contralateral FA & $0.72 \pm 0.03$ & $0.72 \pm 0.03$ & $0.74 \pm 0.04$ & $0.72 \pm 0.04$ & $0.70 \pm 0.05$ & $0.73 \pm 0.04$ \\
Edema ADC $\left(\times 10^{-3} \mathrm{~mm}^{2} / \mathrm{s}\right)$ & $1.15 \pm 0.37$ & $1.13 \pm 0.27$ & $1.35 \pm 0.31$ & $1.33 \pm 0.42$ & $1.57 \pm 0.22$ & $1.53 \pm 0.33$ \\
Ratio of edema ADC & $1.63 \pm 0.56$ & $1.58 \pm 0.46$ & $1.93 \pm 0.50$ & $1.84 \pm 0.62$ & $2.12 \pm 0.30$ & $2.11 \pm 0.47$ \\
Contralateral ADC & $0.71 \pm 0.03$ & $0.72 \pm 0.04$ & $0.71 \pm 0.05$ & $0.74 \pm 0.04$ & $0.74 \pm 0.04$ & $0.73 \pm 0.03$ \\
\hline
\end{tabular}
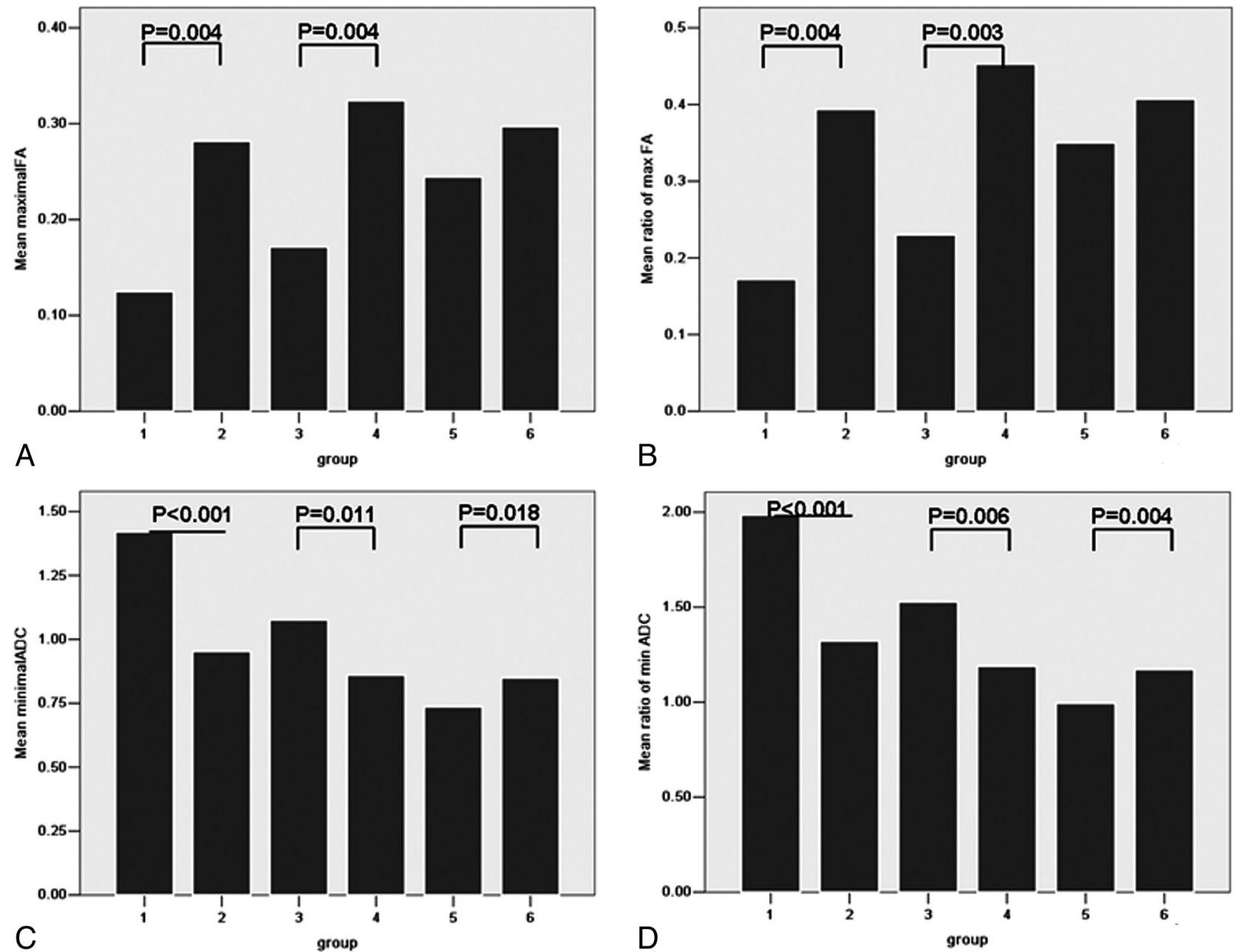

FIG 1. Bar graph of maximal fractional anisotropy $(F A)(A)$, ratio of maximal FA (rmFA) (B), minimal ADC (C), and ratio of minimal $A D C(r m A D C)(D)$ in different groups. $P$ values with statistical significance are shown. Maximal FA and rmFA could differentiate group 1 from group 2 and group 3 from group 4. Minimal ADC (unit: $\times 10^{-3} \mathrm{~mm}^{2} / \mathrm{s}$ ) and rmADC showed statistical significance in groups 1 and 2 , in groups 3 and 4 , and in groups 5 and 6 .

for distinguishing between groups with and without $I D H 1 \mathrm{R} 132 \mathrm{H}$ mutation. The sensitivity and specificity of these parameters were calculated. Sex differences were tested by means of the $\chi^{2}$ test.

\section{RESULTS}

The age and imaging data were normally distributed. The age and imaging parameters in the different groups are shown as mean \pm standard deviation. Sex was shown as the ratio of men to women. The interobserver intraclass correlations were $0.73,0.84,0.65$,
$0.70,0.87$, and 0.86 for the maximal FA, minimal ADC, peritumoral FA, peritumoral ADC, contralateral FA, and contralateral ADC, respectively.

\section{Group 1 and Group 2}

One case in group 2 was recurrent glioma. The differences in age and sex between groups 1 and 2 were not statistically significant (Table 1$)$. The maximal FA $(0.25 \pm 0.16)$ and $\operatorname{rmFA}(0.35 \pm 0.22)$ values in group 2 were higher than those $(0.14 \pm 0.07$ and 
Table 2: Cutoff value, sensitivity, specificity, and AUC of imaging parameters in the differentiation of astroglioma with and without IDH1 R132H mutations

\begin{tabular}{lcccc}
\hline & Cutoff Value & Sensitivity & Specificity & AUC \\
\hline Maximal FA & & & & \\
$\quad$ Groups 1 and 2 & 0.18 & $90.90 \%$ & $96.00 \%$ & 0.92 \\
$\quad$ Groups 3 and 4 & 0.27 & $81.80 \%$ & $83.30 \%$ & 0.80 \\
Ratio of maximal FA & & & & \\
$\quad$ Groups 1 and 2 & 0.25 & $90.90 \%$ & $96.00 \%$ & 0.92 \\
$\quad$ Groups 3 and 4 & 0.37 & $81.80 \%$ & $83.30 \%$ & 0.82 \\
Minimal ADC ( $\left.10^{-3} \mathrm{~mm}^{2} / \mathrm{s}\right)$ & & & & \\
$\quad$ Groups 1 and 2 & 1.07 & $100.00 \%$ & $90.90 \%$ & 0.94 \\
Groups 3 and 4 & 0.99 & $75.00 \%$ & $81.80 \%$ & 0.76 \\
$\quad$ Groups 5 and 6 & 0.81 & $53.50 \%$ & $90.00 \%$ & 0.66 \\
Ratio of minimal ADC & & & & \\
$\quad$ Groups 1 and 2 & 1.47 & $100.00 \%$ & $81.80 \%$ & 0.93 \\
Groups 3 and 4 & 1.31 & $91.70 \%$ & $81.80 \%$ & 0.83 \\
$\quad$ Groups 5 and 6 & 1.10 & $53.50 \%$ & $90.00 \%$ & 0.70 \\
\hline
\end{tabular}
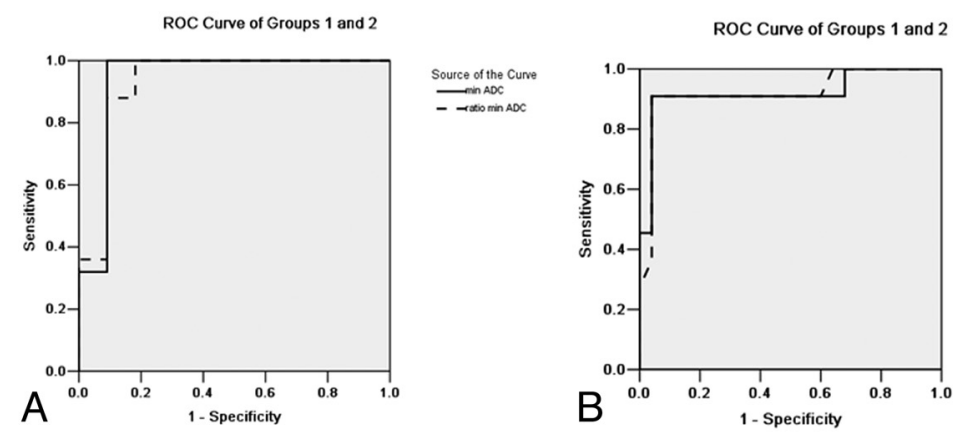

Source of the Curve

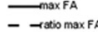

ROC Curve of Groups 3 and 4
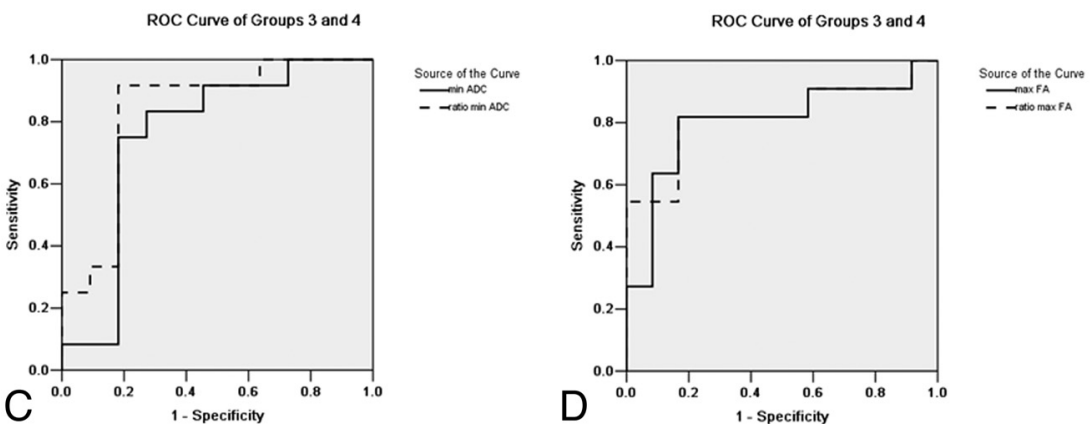

ROC Curve of Groups 5 and 6

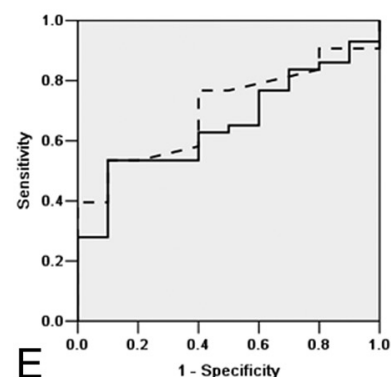

Source of the Curv

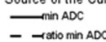

same sensitivity $(90.90 \%)$, specificity (96\%), and area under the curve (AUC) (0.92) (Table 2 and Fig 2). Regarding minimal ADC and rmADC, the values $\left(1.02 \pm 0.29 \times 10^{-3} \mathrm{~mm}^{2} / \mathrm{s}\right.$ and $1.42 \pm$ $0.43)$ in group 2 were lower than those $\left(1.39 \pm 0.34 \times 10^{-3} \mathrm{~mm}^{2} / \mathrm{s}\right.$ and $1.95 \pm$ 0.43 ) in group 1 (both $P<.001$ ) (Figs 1 and 3$)$. When the cutoff values were, respectively, $1.07 \times 10^{-3} \mathrm{~mm}^{2} / \mathrm{s}$ and 1.47 (Fig 2), the sensitivity of both parameters was $100 \%$, and specificities and AUC were, respectively, $90.90 \%, 81.80 \%$, and $0.94,0.93$ (Table 2).

\section{Group 3 and Group 4}

Two cases in group 4 were recurrent glioma. The differences in age and sex between groups 3 and 4 were not statistically significant. The maximal FA value $(0.32 \pm 0.13)$ and $\operatorname{rmFA}(0.45 \pm 0.18)$ in group 4 were higher than those $(0.17 \pm$ 0.10 and $0.23 \pm 0.13)$ in group $3(P=.004$ and 0.003 , respectively) (Figs 1 and 4 ). When the cutoffs were, respectively, 0.27 and 0.37 , the AUC of the rmFA (0.82) was larger than that of maximal FA (0.80) (Fig $2)$. Regarding minimal ADC value and rmADC value, the values $(0.86 \pm 0.21 \times$ $10^{-3} \mathrm{~mm}^{2} / \mathrm{s}$ and $\left.1.19 \pm 0.30\right)$ in group 4 were lower than those $(1.08 \pm 0.15 \times$ $10^{-3} \mathrm{~mm}^{2} / \mathrm{s}$ and $\left.1.53 \pm 0.23\right)$ in group 3 ( $P=.011$ and $P=.006$, respectively) (Figs 1 and 4). The cutoffs for minimal ADC and $\mathrm{rmADC}$ were $0.99 \times 10^{-3} \mathrm{~mm}^{2} / \mathrm{s}$ and 1.31 (Table 2), respectively. The AUC of rmADC (0.83) was larger than that of minimal ADC (0.76) (Fig 2).

\section{Group 5 and Group 6}

In group 5 and group 6 , the differences in age and sex were statistically significant. In group 5, the patients were younger than those in group 6, and male subjects were more common in group 5 ( 8 male to 2 female subjects). There was no statistical significance for maximal FA value or rmFA (Figs 1 and 5). Regarding minimal ADC value and rmADC value, the values $\left(0.74 \pm 0.11 \times 10^{-3} \mathrm{~mm}^{2} / \mathrm{s}\right.$ and $0.99 \pm$ $0.13)$ in group 5 were lower than those $\left(0.85 \pm 0.20 \times 10^{-3} \mathrm{~mm}^{2} / \mathrm{s}\right.$ and $1.17 \pm$ $0.26)$ in group $6(P=.018$ and $P=.004$, respectively) (Figs 1 and 5). The cutoffs

FIG 2. Receiver operating characteristic curves with statistical significance are shown. The area under the curve (AUC) showed a decreasing trend in imaging parameters from grade II to grade IV. Minimal ADC showed the largest AUC in group 1 and group 2, and the second largest parameter was the ratio of minimal ADC (rmADC). In groups 3, 4, 5, and 6, the largest AUC was rmADC.

$0.20 \pm 0.09$ ) in group 1 (both $P=.004$ ) (Fig 1). In receiver operating characteristic curve analysis, when the cutoffs were, respectively, 0.18 and 0.25 (Table 2), the maximal FA and rmFA had the for minimal ADC and rmADC were $0.81 \times 10^{-3} \mathrm{~mm}^{2} / \mathrm{s}$ and 1.10 with the AUC equal to 0.66 and 0.70 , respectively (Table 2 and Fig 2). 


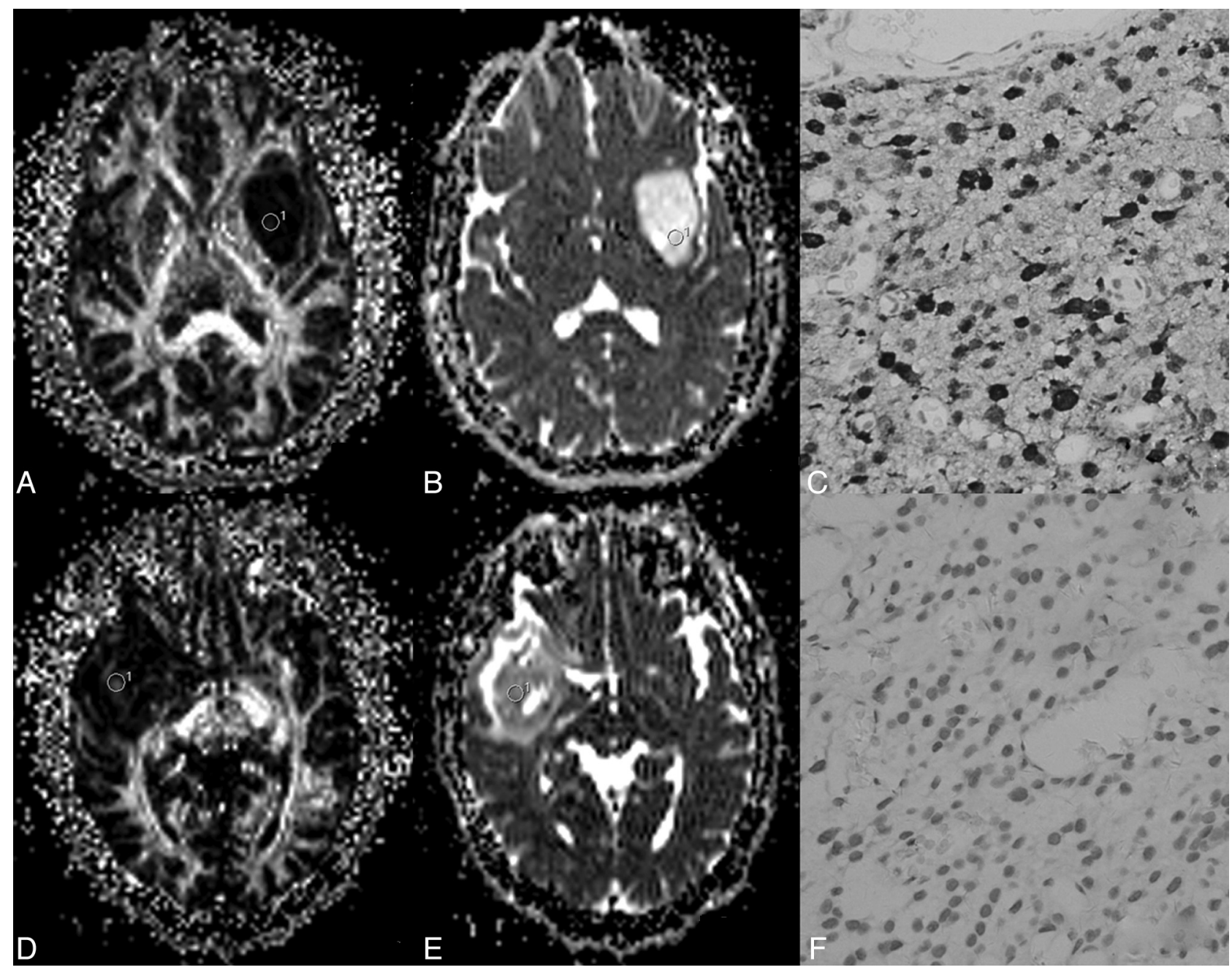

FIG 3. A-C, A 43-year-old man with a grade II astrocytoma and IDHI RI32H antibody examination expressed positively. D-F, A 28-year-old woman with a grade II astrocytoma and IDHI RI32H expressed negatively. In $A$, the fractional anisotropy gray-scale mapping shows a homogeneous low signal; in $D$, a heterogeneous image is shown with a dotted high signal. The signal of ADC mapping in $B$ was higher than that in $E$. $C$ shows diffuse positivity on immunochemistry; $F$ shows negativity on immunochemistry.

Other imaging parameters, including peritumoral FA, ratio of peritumoral FA, contralateral FA, peritumoral ADC, ratio of peritumoral ADC, and contralateral ADC, had no statistical differences between 2 groups with the same WHO grades.

\section{DISCUSSION}

Several studies have suggested that gene expression might be a better predictor of key outcomes than histologic classification. ${ }^{20}$ Thus, noninvasively detecting the genetic characteristics before surgery is important for predicting the outcome and choosing the best therapy. In the current study, we suggested that DTI could be a useful tool for detecting IDH1 R132H mutation in astrogliomas, and, on the basis of receiver operating characteristic curve analysis, we believe that rmADC is the best metric for detecting IDH1 $\mathrm{R} 132 \mathrm{H}$ mutation in astrogliomas.

Diffusion imaging is a method that provides direct insight into the microscopic physical properties of tissues ${ }^{21}$; it has the ability to detect the genetic characteristics of gliomas. Jenkinson et $\mathrm{al}^{22}$ reported that tumors with intact $1 \mathrm{p} / 19 \mathrm{q}$ had higher maximum ADC value on DWI. Khayal et $\mathrm{al}^{15}$ reported that ADC determined from DTI showed promising value for distinguishing oligoastro- cytomas with codeletions in chromosomes $1 \mathrm{p}$ and $19 \mathrm{q}$ from those with intact $1 \mathrm{p} / 19 \mathrm{q}$ chromosomes.

In the present study, we investigated the possibility that ADC and FA from DTI might be used to distinguish astrogliomas with IDH1 R132H mutation from those without mutation. In grade II tumors, minimal ADC had the largest AUC, and the second was rmADC. In grade III and grade IV tumors, rmADC had the largest AUC in the ROC analysis. Thus, we believe that rmADC is the best metric for detecting IDH1 R132H mutation, regardless of the grades. In IDH1 R132H-mutant astrogliomas, maximal FA and rmFA showed an increasing trend from grade II to grade IV, and the minimal $\mathrm{ADC}$ and $\mathrm{rmADC}$ presented a decreasing tendency. However, this tendency was not so distinct in IDH1 wild-type astrogliomas.

The role of IDH1 R132H mutation in tumorigenic processes of glioma remains unclear. The FA and ADC values are largely affected by cellularity and/or vascularity. ${ }^{21-24}$ Zhao et $\mathrm{al}^{25}$ showed that IDH1 appears to function as a tumor suppressor that when mutationally inactivated, contributes to tumorigenesis partially through induction of the HIF-1 pathway. HIF- $1 \alpha$ promotes angiogenesis. This may be one of the reasons for higher FA values 


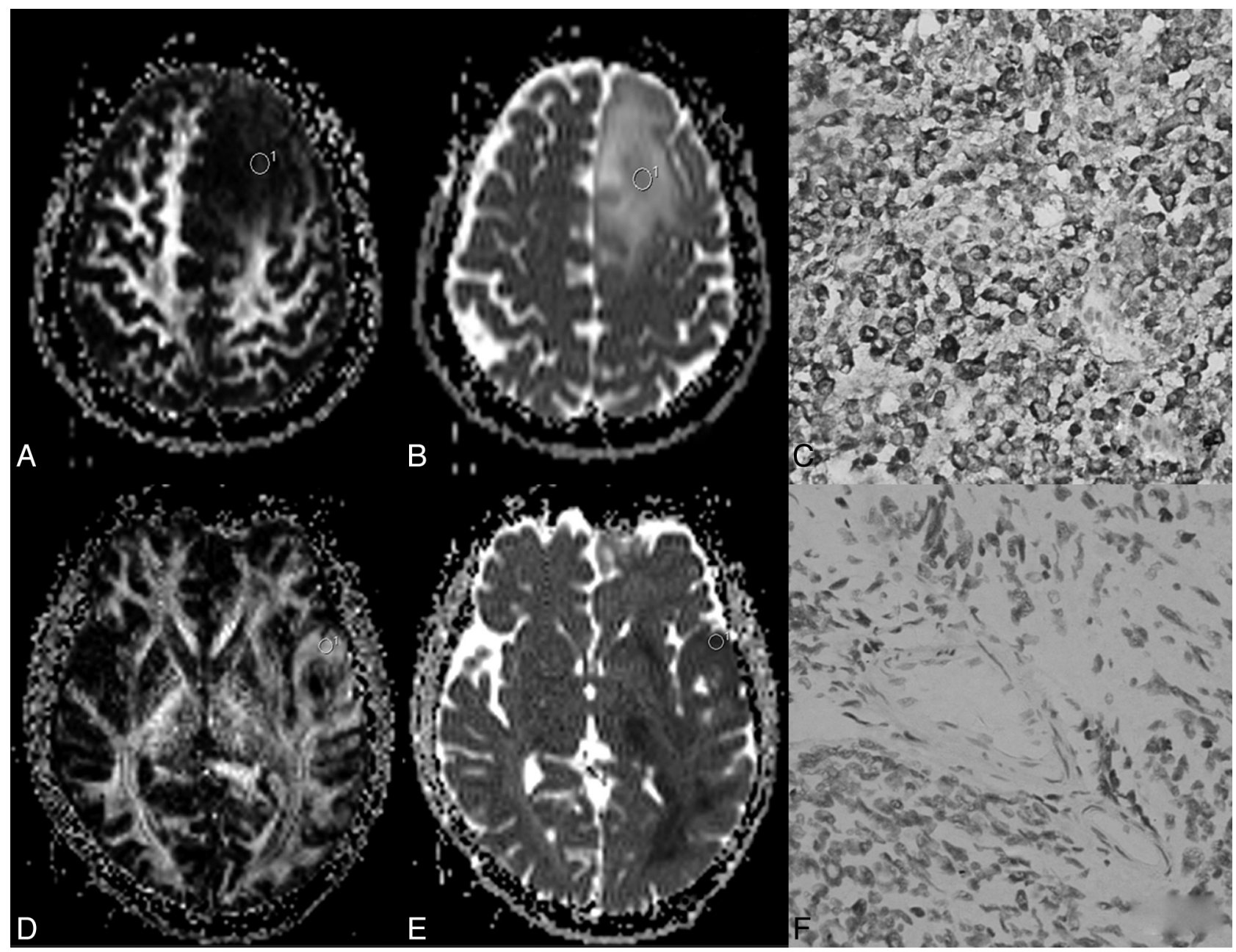

FIG 4. A-C, A 35-year-old woman with a grade III anaplastic astrocytoma and IDHI RI32H antibody examination expressed positively. $D-F, A$ 17-year-old young man with grade III anaplastic astrocytoma and IDHI RI32H antibody examination expressed negatively. In A, fractional anisotropy gray-scale mapping showed a heterogeneous low signal with some iso- and hyper-signals; in $D$, a high signal was seen, except for the middle necrosis area. The signal of ADC mapping in $B$ is higher than that in $E$. $C$ and $F$, Immunochemistry results are shown.

and lower $\mathrm{ADC}$ values in the wild-type group than the mutation group in grade II and III tumors. According to our results, we suggest that future studies should be performed to observe the differences in cellularity and angiogenesis between astrogliomas with and without $I D H 1 \mathrm{R} 132 \mathrm{H}$ mutation.

Furthermore, it is unusual that neither maximal FA nor rmFA demonstrated statistically significant differences in grade IV tumors because they were in grade II and grade III astrogliomas. The minimal ADC and rmADC values in group 5 were less than those in group 6. The sensitivity and specificity of minimal ADC and rmADC were less in grade IV glioblastoma. These phenomena might have 2 reasons. One is that the tumorigenesis in glioblastoma multiforme is a complicated polygene-related process. Many studies have reported that secondary glioblastomas had different subsets of genetic abnormalities from primary glioblastomas. ${ }^{3}$ Even in primary glioblastoma, there are different genes taking part in tumorigenesis. In the present study, grade IV tumors mostly consisted of both primary and secondary glioblastomas, and IDH1 R132H was the only analyzed genetic mutation type. Moreover, $I D H$ mutations are considered as early events in astrocytoma tumorigenesis. ${ }^{26}$ Therefore, we suggest that the prev- alence and role of $I D H 1$ mutations in different grades of astrogliomas result in these phenomena. The small sample size in group 5 might be another reason for these findings.

In some studies, mean FA and ADC have been measured to grade gliomas. However, it is well known that diffuse infiltrating astrocytomas have different grades within the same tumor. ${ }^{27}$ If the diffusion tensor metrics are calculated from whole tumor, there is a theoretic possibility of missing the small foci of highgrade tumors within otherwise low-grade tumors. ${ }^{28}$ If mean FA and ADC were measured in any focal area within tumors, they could not represent the cellular attenuation or the arrangement of cells for the entire tumors. Such a mistake would therefore be magnified. In the present study, maximal FA and minimal ADC were used as diffusion tensor metrics. This method avoided the aforementioned error. In addition, maximal FA and minimal ADC could better reflect the difference between the IDH1$\mathrm{R} 132 \mathrm{H}-\mathrm{mutant}$ and nonmutant groups. This also explains why the maximal FA value in the current study was a little greater than the mean FA values reported in the literature..$^{23,29-31}$

Currently, immunohistochemical analysis and gene sequencing are the main available methods for detecting $I D H$ mutations 


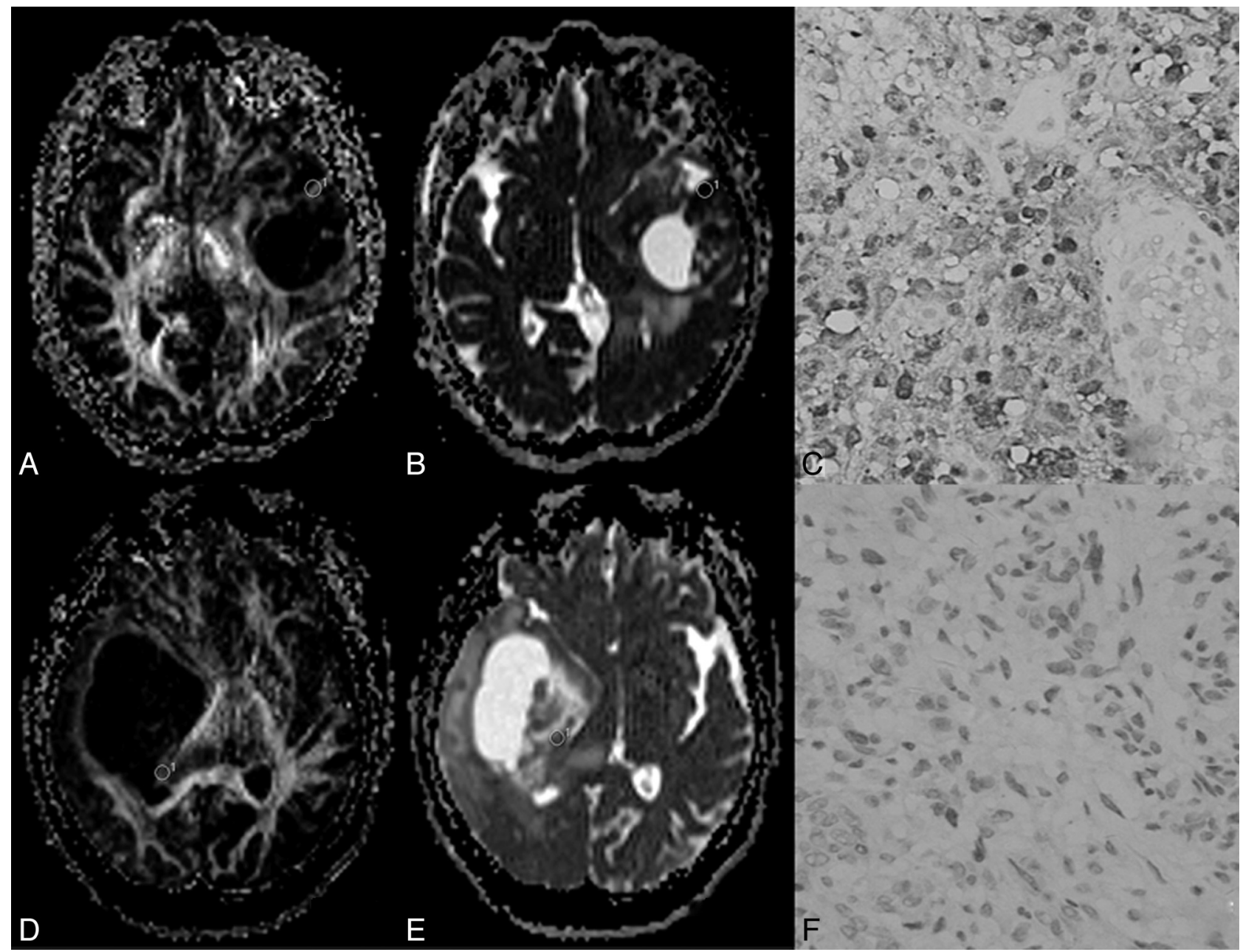

FIG 5. A-C, A 33-year-old woman with a grade IV primary glioblastoma and IDHI RI32H antibody examination expressed positively. $D-F, A$ 48-year-old man with a grade IV primary glioblastoma and IDHI RI32H antibody examination expressed negatively. In $A$ and $D$, fractional anisotropy mappings show heterogeneous images with scattered high signals. The signal of ADC mapping in $B$ is lower than that in $E$. Immunohistochemistry results of these patients are shown.

in gliomas. Gene sequencing is considered the reference standard for detecting all types of $I D H$ mutations. ${ }^{32}$ A monoclonal antibody, H09, has been developed for detecting the most common mutation, IDH1 R132H. The sensitivity and specificity of this antibody for detecting IDH1 R132H reportedly approached $100 \%{ }^{33}$ The accuracy of gene sequencing could be affected by highly contaminated tumor samples or by the quantity of tumor cell in the sample. Capper et $\mathrm{al}^{33}$ reported that $I D H 1$ sequencing missed 8 of 112 cases of IDH1 R132H mutation in the first round of sequencing. The predominant amino acid sequence alteration in $I D H 1 \mathrm{mu}-$ tation was $\mathrm{R} 132 \mathrm{H}$, accounting for $92.7 \%$ of the detected mutations in 1010 cases of WHO grade II and III gliomas, whereas IDH2 mutations were much less common. ${ }^{34}$ Hartmann et al ${ }^{34}$ reported that astrocytoma grades II and III carried only $0.9 \% \mathrm{IDH} 2$ mutations. Because of the high accuracy of monoclonal antibody $\mathrm{H} 09$ and the lower frequency of other IDH1 mutation types and IDH2 mutations in glioma, we chose immunochemical examination as the reference standard for detecting IDH1 R132H mutation.

There were some important limitations to our study. Our study classified astrogliomas from grade II to grade IV, according to pathologic results. The data show some overlap between group
2 and group 3 and between group 4 and group 5. Therefore, imaging techniques with high accuracy to grade astrogliomas must be combined with this technique in the future. Although the patients with brain stem or thalamic astrogliomas would benefit most from IDH1 status by avoiding biopsy, all the cases included in the current study were lobar tumors. Therefore, the results in this study should be applied carefully to particular location astrogliomas. Because this was a retrospective study, there was no precise point-to-point biopsy guided by maximal FA and minimal ADC information. Thus, the correlation of maximal FA and minimal ADC with histopathologic change could not be assessed, which is especially important for the evaluation of grades III and IV tumors because these tumors display more heterogeneity. Future prospective studies with point-to-point biopsy design will be necessary to verify our findings of optimal parameters for accurate preoperative detection of IDH1 R132H mutation.

\section{CONCLUSIONS}

We demonstrate that DTI metrics, including maximal FA, rmFA, minimal ADC, and rmADC from the tumor body have potential ability to distinguish astrogliomas with $I D H 1 \mathrm{R} 132 \mathrm{H}$ mutation 
from those without mutation in grade II and grade III tumors. Minimal ADC and rmADC in grade IV tumors are helpful to differentiate glioblastomas with IDH1 R132H mutation from those without mutation. The rmADC is the best metric for the noninvasive detection of such mutation across different tumor grades. Nonetheless, these results are preliminary, and further investigation with histologic validation will be necessary to prove the difference between astrogliomas with IDH1 R132H mutation and those without mutation.

\section{REFERENCES}

1. Parsons DW, Jones S, Zhang X, et al. An integrated genomic analysis of human glioblastoma multiforme. Science 2008;321:1807-12

2. Yan H, Parsons DW, Jin G, et al. IDH1 and IDH2 mutations in gliomas. N Engl J Med 2009;360:765-73

3. Nikiforova MN, Hamilton RL. Molecular diagnostics of gliomas. Arch Pathol Lab Med 2011;135:558-68

4. Horbinski C, Kofler J, Kelly LM, et al. Diagnostic use of IDH1/2 mutation analysis in routine clinical testing of formalin-fixed, paraffin-embedded glioma tissues. J Neuropathol Exp Neurol 2009;68: 1319-25

5. Capper D, Sahm F, Hartmann C, et al. Application of mutant IDH1 antibody to differentiate diffuse glioma from nonneoplastic central nervous system lesions and therapy-induced changes. Am J Surg Pathol 2010;34:1199-204

6. Juratli TA, Kirsch M, Robel K, et al. IDH mutations as an early and consistent marker in low-grade astrocytomas WHO grade II and their consecutive secondary high-grade gliomas. NeuroOncol 2012; 108:403-10

7. SongTao Q, Lei Y, Si G, et al. IDH mutations predict longer survival and response to temozolomide in secondary glioblastoma. Cancer Sci 2012;103:269-73

8. Loussouarn D, Le Loupp AG, Frenel JS, et al. Comparison of immunohistochemistry, DNA sequencing and allele-specific PCR for the detection of IDH1 mutations in gliomas. Int J Oncol 2012;40: 2058-62

9. Metellus P, Coulibaly B, Colin C, et al. Absence of IDH mutation identifies a novel radiologic and molecular subtype of WHO grade II gliomas with dismal prognosis. Acta Neuropathol 2010;120: $719-29$

10. Dang L, White DW, Gross S, et al. Cancer-associated IDH1 mutations produce 2-hydroxyglutarate. Nature 2009;462:739-44

11. Ward PS, Patel J, Wise DR, et al. The common feature of leukemiaassociated IDH1 and IDH2 mutations is a neomorphic enzyme activity converting alpha-ketoglutarate to 2-hydroxyglutarate. Cancer Cell 2010;17:225-34

12. Capper D, Simon M, Langhans CD, et al. 2-Hydroxyglutarate concentration in serum from patients with gliomas does not correlate with IDH1/2 mutation status or tumor size. Int J Cancer 2012; 131:766-68

13. Choi C, Ganji SK, DeBerardinis RJ, et al. 2-hydroxyglutarate detection by magnetic resonance spectroscopy in IDH-mutated patients with gliomas. Nat Med 2012;18:624-29

14. Pope WB, Prins RM, Albert Thomas M, et al. Non-invasive detection of 2-hydroxyglutarate and other metabolites in IDH1 mutant glioma patients using magnetic resonance spectroscopy. J NeuroOncol 2012;107:197-205

15. Khayal IS, Vandenberg SR, Smith KJ, et al. MRI apparent diffusion coefficient reflects histopathologic subtype, axonal disruption, and tumor fraction in diffuse-type grade II gliomas. NeuroOncol 2011; 13:1192-201

16. Louis DN, Ohgaki H, Wiestler OD, et al. The 2007 WHO classification of tumours of the central nervous system. Acta Neuropathol 2007;114:97-109

17. Takano S, Tian W, Matsuda M, et al. Detection of IDH1 mutation in human gliomas: comparison of immunohistochemistry and sequencing. Brain Tumor Pathol 2011;28:115-23

18. Khalid L, Carone M, Dumrongpisutikul N, et al. Imaging characteristics of oligodendrogliomas that predict grade. AJNR Am J Neuroradiol 2012;33:852-57

19. Brander A, Kataja A, Saastamoinen A, et al. Diffusion tensor imaging of the brain in a healthy adult population: normative values and measurement reproducibility at $3 \mathrm{~T}$ and $1.5 \mathrm{~T}$. Acta Radiol 2010;51:800-07

20. Nutt CL, Mani DR, Betensky RA, et al. Gene expression-based classification of malignant gliomas correlates better with survival than histological classification. Cancer Res 2003;63:1602-07

21. Le Bihan D. Looking into the functional architecture of the brain with diffusion MRI. Nat Rev Neurosci 2003;4:469-80

22. Jenkinson MD, Smith TS, Brodbelt AR, et al. Apparent diffusion coefficients in oligodendroglial tumors characterized by genotype. J Magn Reson Imaging 2007;26:1405-12

23. Beppu T, Inoue T, Shibata $Y$, et al. Measurement of fractional anisotropy using diffusion tensor MRI in supratentorial astrocytic tumors. J NeuroOncol 2003;63:109-16

24. Mikkelsen T. Predicting survival in glioblastomas using diffusion tensor imaging metrics. J Magn Reson Imaging 2010;32:788-95

25. Zhao S, Lin Y, Xu W, et al. Glioma-derived mutations in IDH1 dominantly inhibit IDH1 catalytic activity and induce HIF-1alpha. Science 2009;324:261-65

26. Liu Y, Jiang W, Liu J, et al. IDH1 mutations inhibit multiple $\boldsymbol{\alpha}$-ketoglutarate-dependent dioxygenase activities in astroglioma. $J$ Neurooncol 2012;109:253-60

27. Graham DI, Lantos PL. Greenfield's Neuropathology. London: Hodder Arnold Publisher; 2002:767-1052

28. Jolapara M, Patro SN, Kesavadas C, et al. Can diffusion tensor metrics help in preoperative grading of diffusely infiltrating astrocytomas? A retrospective study of $\mathbf{3 6}$ cases. Neuroradiology 2011;53: 63-68

29. Byrnes TJ, Barrick TR, Bell BA, et al. Diffusion tensor imaging discriminates between glioblastoma and cerebral metastases in vivo. NMR Biomed. 2011;24:54-60

30. Jakab A, Molnár P, Emri M, et al. Glioma grade assessment by using histogram analysis of diffusion tensor imaging-derived maps. $\mathrm{Neu}$ roradiology 2011;53:483-91

31. Zou QG, Xu HB, Liu F, et al. In the assessment of supratentorial glioma grade: the combined role of multivoxel proton MR spectroscopy and diffusion tensor imaging. Clin Radiol 2011;66:953-60

32. Gupta R, Webb-Myers R, Flanagan S, et al. Isocitrate dehydrogenase mutations in diffuse gliomas: clinical and aetiological implications. J Clin Pathol 2011;64:835-44

33. Capper D, Weissert S, Balss J, et al. Characterization of R132H mutation-specific IDH1 antibody binding in brain tumors. Brain Pathol 2010;20:245-54

34. Hartmann C, Meyer J, Balss J, et al. Type and frequency of IDH1 and IDH2 mutations are related to astrocytic and oligodendroglial differentiation and age: a study of 1,010 diffuse gliomas. Acta Neuropathol 2009;118:469-74 